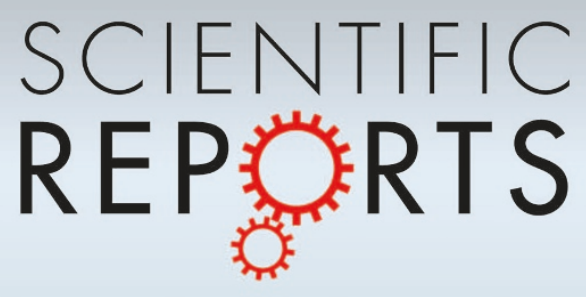

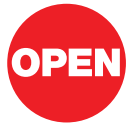

SUBJECT AREAS:

ELECTRONIC PROPERTIES

AND MATERIALS

THEORY AND COMPUTATION

SURFACES, INTERFACES AND

THIN FILMS

NANOSCALE MATERIALS

Received

7 November 2012

Accepted

31 December 2012

Published

12 February 2013

Correspondence and requests for materials should be addressed to

U.S. ludo.

schwingenschlogl@

kaust.edu.sa)

\section{Quantum capacitance of an ultrathin topological insulator film in a magnetic field}

\author{
M. Tahir' ${ }^{1}$ K. Sabeeh ${ }^{2} \&$ U. Schwingenschlögl' \\ 'PSE Division, KAUST, Thuwal 23955-6900, Kingdom of Saudi Arabia, ${ }^{2}$ Department of Physics, Quaid-i-Azam University, \\ Islamabad 45320 Pakistan.
}

We present a theoretical study of the quantum magnetocapacitance of an ultrathin topological insulator film in an external magnetic field. The study is undertaken to investigate the interplay of the Zeeman interaction with the hybridization between the upper and lower surfaces of the thin film. Determining the density of states, we find that the electron-hole symmetry is broken when the Zeeman and hybridization energies are varied relative to each other. This leads to a change in the character of the magnetocapacitance at the charge neutrality point. We further show that in the presence of both Zeeman interaction and hybridization the magnetocapacitance exhibits beating at low and splitting of the Shubnikov de Haas oscillations at high perpendicular magnetic field. In addition, we address the crossover from perpendicular to parallel magnetic field and find consistency with recent experimental data.

. opological insulators (TIs) form a new class of compounds with strong spin-orbit interaction ${ }^{1-8}$. These materials possess an energy gap in the bulk (like insulators) and conducting electronic states at the surface (like metals). The nature of the surface states depends on the dimensions of the $\mathrm{TI}^{9-16}$ : Two-dimensional surface states have been revealed in transport experiments for three-dimensional TIs. The charge carriers in such surface states form a two-dimensional gas of Dirac fermions with a single Dirac cone energy spectrum. Dirac fermions have been observed in angle resolved photo-emission experiments and quantization of their Landau levels (LLs) has been confirmed by scanning tunneling spectroscopy ${ }^{17-21}$. TI thin films provide a new degree of freedom which is the thickness of the film. Effects of the crossover from $3 \mathrm{D}$ to $2 \mathrm{D}$ topological surface states as well as hybridization of the states at the two surfaces of the thin film have been studied for varying film thicknesses and found to become important when the thickness is less than about $5 \mathrm{~nm}^{22-26}$. The Dirac fermions in TIs share several features with those of graphene ${ }^{27,28}$. However, there are important differences between graphene and the surface states of a TI. Unlike graphene, there is an odd number of not spin degenerate Dirac points at any surface. In addition, TIs exhibit a unique spin-momentum locking in the surface states.

Observation of magnetic quantum oscillations, Shubnikov de Haas (SdH) and de Haas van Alphen ${ }^{29,30}$, in the transport experiments has resulted in theoretical work on magnetization ${ }^{29}$ and Berry phase ${ }^{30,31}$ features of TI surface Dirac fermions. Furthermore, much attention is paid to magnetically doped TIs due to their exceptional properties and potential spintronics applications ${ }^{10,24}$. In this context, capacitance measurements are among the most important tools for studying the electronic properties. They can be effectively used to probe the thermodynamic density of states (DOS). Although the focus in TI research has been on transport properties, insight into the fundamental electronic properties and device physics still calls for knowledge about the capacitance-voltage (CV) characteristics of the system. Results have been reported for carbon nanotubes, graphene nanoribbons, and mono- and bilayer graphene systems ${ }^{32-37}$. In view of this, attention is now being paid to electrostatic properties, such as the magnetocapacitance of TI thin film devices. Furthermore, for improve the performance of field effect transistors $^{38}$, the potential of TI thin films as channel materials is creating much excitement. This is due to the excellent intrinsic transport features as well as the possibility of patterning device structures within top-down lithographical techniques. The present work aims at determining the combined effects of the Zeeman interaction and hybridization on the magnetocapacitance of a TI thin film device.

\section{Results}

We consider Dirac fermions on the surface of a 3D TI thin film which we take to be in the $x y$-plane. There is an external magnetic field perpendicular to the thin film and we take into account hybridization between the upper 
and lower surfaces. The two-dimensional Hamiltonian for Dirac fermions in a magnetic field with hybridization is given by ${ }^{24,27,28}$

$$
H=v\left(\sigma_{x} \pi_{y}-\tau_{z} \sigma_{y} \pi_{x}\right)+\left(\tau_{z} \Delta_{z}+\Delta_{h}\right) \sigma_{z} .
$$

Here, $\sigma_{x}$ and $\sigma_{y}$ are Pauli matrices that operate on a combined space of spin and surface degrees of freedom, $\tau_{z}=+/-$ denotes symmetric/antisymmetric combinations of the upper and lower surfaces of the TI, $v$ denotes the Fermi velocity of the Dirac fermions, $\boldsymbol{\pi}=\mathbf{p}+$ $e \mathrm{~A} / c$ is the two-dimensional canonical momentum with vector potential $\mathrm{A}$, and $c$ is the speed of light. Moreover, we have the Zeeman energy $\Delta_{z}=\frac{1}{2} g \mu_{B} B$, the effective Lande factor $g$, the Bohr magneton $\mu_{B}$, and the hybridization matrix element $\Delta_{h}$, which reflects the hybridization between the upper and lower surfaces of the TI. We employ the Landau gauge and express the vector potential as $\mathbf{A}=(0, B x, 0)$. The LL energies then are given by

$$
\begin{aligned}
& E_{0}^{\tau_{z}}=-\left(\Delta_{z}+\tau_{z} \Delta_{h}\right) \\
& E_{n, \lambda}^{\tau_{z}}=\lambda \sqrt{2 n \hbar^{2} \omega^{2}+\left(\Delta_{z}+\tau_{z} \Delta_{h}\right)^{2}}, \quad n \neq 0
\end{aligned}
$$

with $\lambda=+/-$ for electron/hole bands. Moreover, $\omega=v \sqrt{e B / \hbar}$ is the cyclotron frequency of the Dirac fermions and $n$ is an integer. The main point to note about the energy spectrum, which will be crucial to the following discussion, is: The splitting of the energy levels depends on both the Zeeman interaction and the hybridization between the upper and lower surfaces. If either of these two mechanisms is absent, no splitting will occur. Further, the energy spectrum is electron-hole symmetric in the absence of the Zeeman interaction, with a hybridization gap. We note that angular resolved photoelectron spectroscopy finds no electron-hole symmetry even in the absence of both Zeeman interaction and hybridization, reflecting a quadratic term in the Hamiltonian ${ }^{39,40}$. However, in our surface Hamiltonian in Eq. (2) no quadratic term is present as it can be neglected when the system is doped such that the Dirac point comes close to the charge neutrality point, which is the focus of our work. In the presence of both Zeeman interaction and hybridization, the $n=$ 0 LL explicitly shows a quantum phase transition. This will be important in the discussion of the DOS and the capacitance results.

We consider a gated TI device in which the capacitor is formed between the gate and the TI thin film. The magnetocapacitance of the device represents the charge response in the channel as the channel potential is varied. In conventional devices, the magnetocapacitance $C_{Q}$ is large and can be ignored. On the contrary, in low dimensional devices, such as 2D electron gases, graphene, and future TI devices, it is the dominant capacitive contribution and therefore an important quantity in the design of nanoscale devices. The central expression for the quantum capacitance is ${ }^{36}$

$$
C_{Q}=\frac{e \partial Q}{\partial \varepsilon_{F}}=\frac{e^{2} \partial n_{e}}{\partial \varepsilon_{F}}=e^{2} D_{T}(B)
$$

where $n_{e}$ is the carrier concentration and $\varepsilon_{F}$ the Fermi energy. The temperature dependent DOS at a finite magnetic field, $D_{T}(B)$, is obtained from the relation

$$
D_{T}(B)=\frac{\partial n_{e}}{\partial \varepsilon_{F}}=\int_{0}^{\infty} d \varepsilon \frac{\partial f\left(\varepsilon-\varepsilon_{F}\right)}{\partial \varepsilon_{F}} D(\varepsilon)
$$

with the Fermi Dirac distribution $f$. In the limit of zero temperature we have $D_{T}(B)=D\left(\varepsilon_{F}\right)$ and

$$
D\left(\varepsilon_{F}\right)=\frac{1}{2 \pi l^{2}} \sum_{n, \tau_{z}, \lambda} \delta\left(\varepsilon_{F}-E_{n, \lambda}^{\tau_{z}}\right) .
$$

Assuming a Gaussian broadening of the LLs, the DOS per unit area is given by

$$
D\left(\varepsilon_{F}\right)=\frac{1}{2 \pi l^{2}} \sum_{n, \tau_{z}, \lambda} \frac{1}{\Gamma \sqrt{2 \pi}} \exp \left[-\frac{\left(\varepsilon_{F}-E_{n, \lambda}^{\tau_{z}}\right)^{2}}{2 \Gamma^{2}}\right],
$$

where $\Gamma$ is the width of the Gaussian distribution (zero shift). For $n=$ 0 we have

$$
D_{n=0}\left(\varepsilon_{F}\right)=\frac{1}{2 \pi l^{2}} \sum_{\tau_{z}} \frac{1}{\Gamma \sqrt{2 \pi}} \exp \left[-\frac{\left(\varepsilon_{F}-E_{0}^{\tau_{z}}\right)^{2}}{2 \Gamma^{2}}\right],
$$

where $E_{0}^{\tau_{z}}=-\left(\Delta_{z}+\tau_{z} \Delta_{h}\right)$. Combining Eqs. (6) and (7), we obtain

$$
\begin{aligned}
D\left(\varepsilon_{F}\right)= & \frac{1}{2 \pi l^{2}}\left\{\sum_{\tau_{z}} \frac{1}{\Gamma \sqrt{2 \pi}} \exp \left[-\frac{\left(\varepsilon_{F}-E_{0}^{\tau_{z}}\right)^{2}}{2 \Gamma^{2}}\right]\right. \\
& \left.+\sum_{\tau_{z}, \lambda, n=1}^{\infty} \frac{1}{\Gamma \sqrt{2 \pi}} \exp \left[-\frac{\left(\varepsilon_{F}-E_{n, \lambda}^{\tau_{z}}\right)^{2}}{2 \Gamma^{2}}\right]\right\} .
\end{aligned}
$$

At the charge neutrality point $\left(\varepsilon_{F}=0\right)$ the result is

$$
\begin{aligned}
D\left(\varepsilon_{F}=0\right)= & \frac{1}{2 \pi l^{2}} \sum_{\tau_{z}} \frac{1}{\Gamma \sqrt{2 \pi}} \exp \left[-\frac{\left(\Delta_{z}+\tau_{z} \Delta_{h}\right)^{2}}{2 \Gamma^{2}}\right] \\
& \left.\left\{1+2 \sum_{n=1}^{\infty} \exp \left[-\frac{2 n \hbar^{2} \omega^{2}}{2 \Gamma^{2}}\right]\right\}\right)
\end{aligned}
$$

which can be written as

$D\left(\varepsilon_{F}=0\right)=\left(\frac{\Gamma}{\left(V_{F} \hbar\right)^{2} \pi \sqrt{2 \pi}} \sum_{\tau_{z}} \exp \left[-\frac{\left(\Delta_{z}+\tau_{z} \Delta_{h}\right)^{2}}{2 \Gamma^{2}}\right]\left\{\frac{\chi}{\tanh \chi}\right\}\right)(10)$ where $\chi=\hbar^{2} \omega^{2} / 2 \Gamma^{2}$. Equation (10) reduces to the result reported in Ref. 36 in the absence of Zeeman interaction and hybridization.

The magnetocapacitance $C_{Q}$ is plotted in Figs. 1 and 2 as a function of the Fermi energy (i.e., of the gate voltage). The following parameters are employed ${ }^{22,30,41-43}: g=60, B=3 \mathrm{~T}, \Delta_{z}=5 \mathrm{meV}$, and $\Delta_{h}=$ $3 \mathrm{meV}$. To obtain analytical results, we choose a constant level width of $\Gamma=0.3 \mathrm{meV}$. We are interested in changes of the character at $\varepsilon_{F}=$ 0 on variation of the Zeeman interaction and hybridization relative to each other. It must be noted that the $n=0$ LL here plays the most important role. Figure 1 shows that $C_{Q}$ is zero at $\varepsilon_{F}=0$. This occurs because the $n=0 \mathrm{LL}$ splits only when the hybridization does not vanish. The $n=0 \mathrm{LL}$ splits into one electron and one hole level, which reflects a metal to insulator transition caused by the

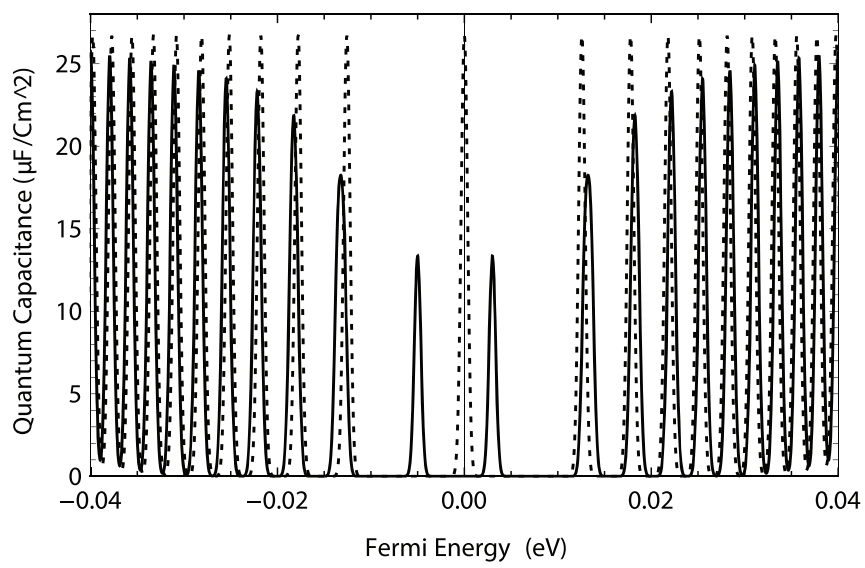

Figure $1 \mid$ Quantum capacitance as a function of the Fermi energy at $T=0 \mathrm{~K}, B=3 \mathrm{~T}$, and Zeeman energy $0 \mathrm{meV}$. The hybridization energy is $0 \mathrm{meV}$ (dotted lines) and $4 \mathrm{meV}$ (solid lines), respectively. 


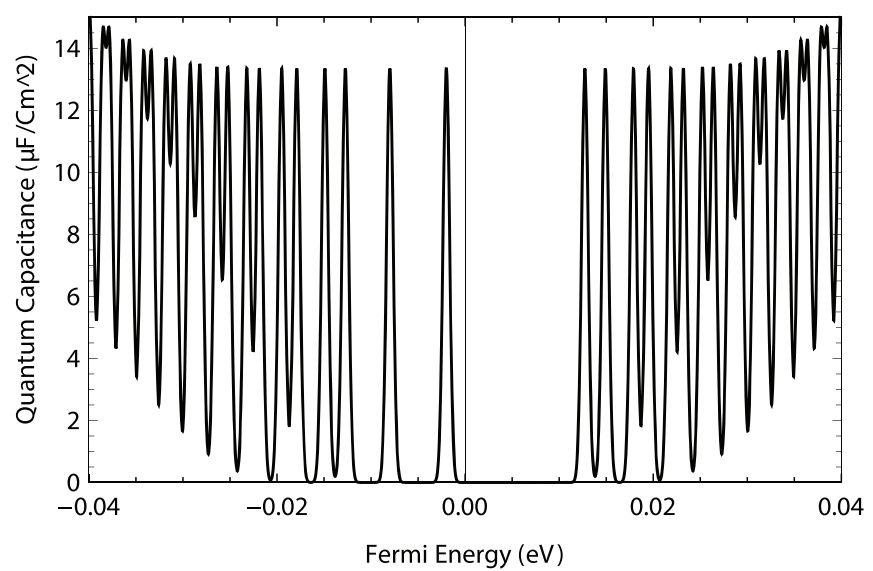

Figure $2 \mid$ Quantum capacitance as a function of the Fermi energy at $T=0 \mathrm{~K}, B=3 \mathrm{~T}$, Zeeman energy $5 \mathrm{meV}$, and hybridization energy $3 \mathrm{meV}$.

hybridization (for $\Delta_{z}=0$ ). The states are electron-hole symmetric at this stage. $C_{Q}$ can be tuned from a minimum to a maximum when the Zeeman energy is increased by changing the external magnetic field. For $\Delta_{z}>\Delta_{h}$ both $n=0$ sublevels are located in the hole region, which breaks the electron-hole symmetry, see Fig. 2, and represents the trivial to non-trivial topological insulator phase transition. To observe the splitting, the broadening of the LLs must be less than the hybridization energy. We note that not only the $n=0$ LL but all LLs are split into two sublevels.

In Fig. 3 we address the $\mathrm{SdH}$ oscillations in $C_{Q}$. The following parameters are used: $\Gamma=0.3 \mathrm{meV}, T=0 \mathrm{~K}, n_{e}=4 \times 10^{15} \mathrm{~m}^{-2}, v$ $=3 \times 10^{5} \mathrm{~ms}^{-1}$, and $\Delta_{h}=4 \mathrm{meV}$. For low and high magnetic field we observe a beating pattern and a splitting of the $\mathrm{SdH}$ oscillations, respectively. Both occurs due to interference of the $\mathrm{SdH}$ oscillations at two different frequencies and therefore is a consequence of the splitting of the LLs for finite Zeeman interaction and hybridization. The beating pattern vanishes once the Zeeman interaction dominates the hybridization. For the chosen parameters this occurs at a magnetic field of about $1.5 \mathrm{~T}$. Above this value we find a well resolved splitting of the $\mathrm{SdH}$ oscillations in Fig. 3. A similar beating pattern and splitting of $\mathrm{SdH}$ oscillations in the longitudinal resistivity is seen in Ref. 44, where it occurs because of splitting of the LLs as a result of a broken inversion symmetry due to substrate effects. The sample investigated in this reference is thick and hybridization effects are ruled out.

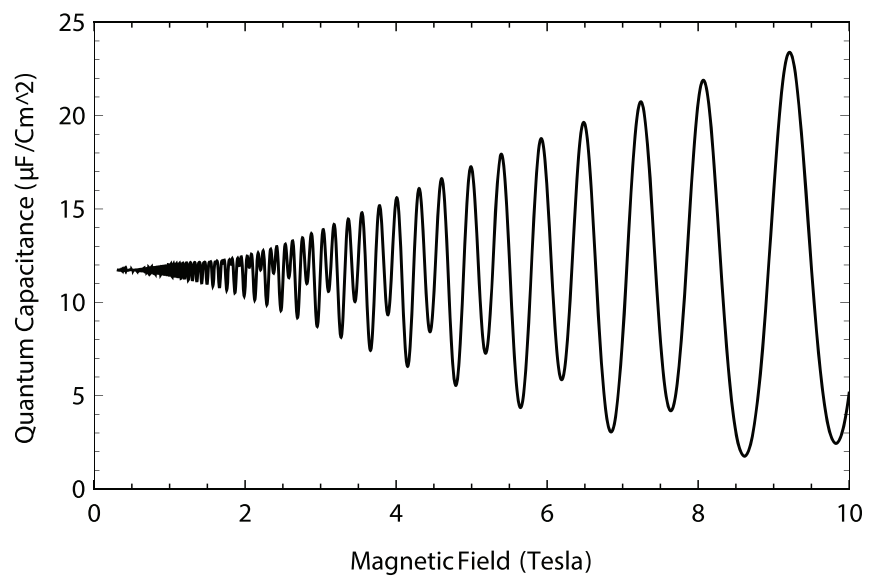

Figure $3 \mid$ Quantum capacitance as a function of the magnetic field at $T=0 \mathrm{~K}$ and hybridization energy $4 \mathrm{meV}$.
In order to understand the origin of the beating pattern and splitting of the $\mathrm{SdH}$ oscillations quantatively, we employ the Poisson summation formula

$\frac{1}{2} F(0)+\sum_{k=1}^{\infty} F(k)=\int_{0}^{\infty} F(n) d n+2 \sum_{k=1}^{\infty}(-1)^{k} \int_{0}^{\infty} F(n) \cos [2 \pi k n] d n(11)$

to arrive at the following expression for the DOS at zero temperature which incorporates the contributions of the $n \geq 1$ LLs:

$$
\begin{aligned}
D\left(\varepsilon_{F}\right)= & D_{0}\left\{1+2 \sum_{\tau_{z}, k=1}^{\infty}(-1)^{k} \exp \left[-\left(\frac{2 \pi k \varepsilon_{F} \sqrt{2} \Gamma}{\hbar^{2} \omega^{2}}\right)^{2}\right]\right. \\
& \left.\cos \left[\frac{2 \pi k}{\hbar^{2} \omega^{2}}\left(\varepsilon_{F}^{2}-\left(\Delta_{z}+\tau_{z} \Delta_{h}\right)^{2}\right)\right]\right\}
\end{aligned}
$$

Here, $D_{0}=\left|\varepsilon_{F}\right| / \pi \hbar^{2} v^{2}$ is the zero magnetic field DOS. For $\Gamma \gg \hbar \omega$, it is sufficient to retain only the first order term $(k=1)$, since the higher order terms are highly damped. From Eq. (12) we can confirm that the beating pattern is due to the interference of waves with two different frequencies. The oscillatory part of the $k=1$ term in the sum for $\tau_{z}=+/-$ is

$\cos \left[\frac{2 \pi}{\hbar^{2} \omega^{2}}\left(\varepsilon_{F}^{2}-\left(\Delta_{z}+\Delta_{h}\right)^{2}\right)\right]+\cos \left[\frac{2 \pi}{\hbar^{2} \omega^{2}}\left(\varepsilon_{F}^{2}-\left(\Delta_{z}-\Delta_{h}\right)^{2}\right)\right]$

and can be expressed as

$$
2 \cos \left[\frac{2 \pi}{\hbar^{2} \omega^{2}}\left(\varepsilon_{F}^{2}-\Delta_{z}^{2}-\Delta_{h}^{2}\right)\right] \cos \left[\frac{4 \pi}{\hbar^{2} \omega^{2}} \Delta_{z} \Delta_{h}\right] .
$$

This represents a wave of higher frequency whose amplitude oscillates at a lower frequency, giving rise to the beating pattern, because $\Delta_{z} \Delta_{h}<<\varepsilon_{F}$. The amplitude of the $\mathrm{SdH}$ oscillations is modulated by $\cos \left[\frac{4 \pi}{\hbar^{2} \omega^{2}} \Delta_{z} \Delta_{h}\right]$ and nodes occur at $\frac{4 \Delta_{z} \Delta_{h}}{\hbar^{2} \omega^{2}}= \pm 0.5, \pm 1.5, \ldots$, where the modulating cosine vanishes. We also note that the amplitude modulation given by the term $\cos \left[\frac{4 \pi}{\hbar^{2} \omega^{2}} \Delta_{z} \Delta_{h}\right]$ occurs when both Zeeman interaction and hybridization are present in the system. Moreover, the threshold magnetic field where beating is seen depends on the strenghts of the Zeeman interaction and hybridization. For magnetic fields down to about $1.5 \mathrm{~T}$ the beating persists. Above this value it is quenched to show splitting of the SdH oscillations. As mentioned before this finding is in agreement with the experimental work in Ref. 44.

\section{Discussion}

Topological systems are typically doped away from the charge neutrality point. In addition, the bulk usually dominates the surface contributions. However, thin films of topological insulators make it possible to access the surface contributions only ${ }^{22,41-43}$, typically when the thickness is below $5 \mathrm{~nm}$. In this context, we compare our results with the experimental observations for the quantum magnetocapacitance $^{45}$. The latter authors study films that are thick enough that hybridization between the top and bottom surfaces can be ignored (10 $\mathrm{nm} \mathrm{Bi}{ }_{2} \mathrm{Se}_{3}$ samples). To compare their results for a tilted magnetic field, we next consider this situation and explore the crossover from external perpendicular to parallel (in-plane) magnetic field on the quantum capacitance. For a tilt angle $0^{\circ} \leq \theta \leq 90^{\circ}$ with the normal of the film, the magnetic field is given by $(B \sin \theta, 0, B \cos$ $\theta)$. To describe the effects of the tilting we start from the two-dimensional Hamiltonian

$$
H=v \tau_{z}\left(\sigma_{x} \pi_{y}-\sigma_{y} \pi_{x}\right)
$$

where $A=(0, x B \cos \theta-z B \sin \theta, 0)$ and $z$ is the thickness of the film. The LL energies are obtained as 


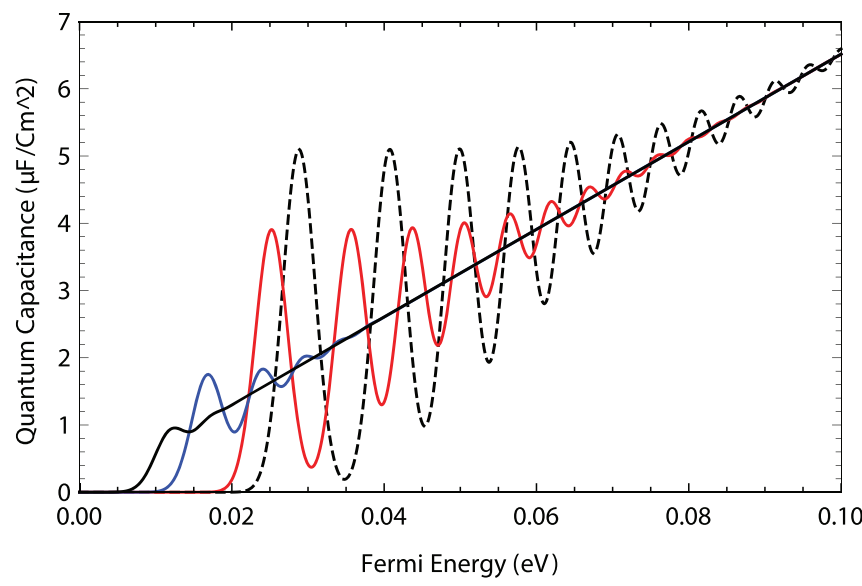

Figure $4 \mid$ Quantum capacitance as a function of the Fermi energy for different tilt angles of the magnetic field: $0^{\circ}$ (dashed lines), $40^{\circ}$ (red solid lines), $70^{\circ}$ (blue solid lines), and $80^{\circ}$ (black solid lines). We use $B=7 \mathrm{~T}$ and $\Gamma=25 \mathrm{~K}$.

$$
E_{n, \lambda}=\lambda \sqrt{2 n} \hbar \omega_{t},
$$

with $\omega_{t}=v \sqrt{e B \cos \theta / \hbar}$. Note the dependence of the LLs on the tilt angle $\theta$ as compared to Eq. (2).

Following the same procedure as before, we employ the energy spectrum to compute the quantum capacitance, which is plotted in Fig. 4 as a function of the Fermi energy (gate voltage) for different tilt angles. We focus on the broadening of the LLs and the corresponding effects on the SdH oscillations. We fix $B=7 \mathrm{~T}$. The SdH oscillations are suppressed as we increase the tilt angle, since we are decreasing the perpendicular component of the magnetic field. For $\theta \rightarrow 90^{\circ}$, when the magnetic field is almost completely aligned with the film, the $\mathrm{SdH}$ oscillations are washed out. The broadening of the LLs generally depends on the magnetic field strength, LL index, and scattering parameters. This requires a self consistent calculation, which usually is performed numerically. In order to carry out a tractable analytical calculation we choose a constant level width of $\Gamma=25 \mathrm{~K}$. The results in Fig. 4 are consistent with the experimental Fig. 4 in Ref. 45.

An experimental investigation of transport arising from the surface states in TIs is hindered by the bulk contributions due to naturally occurring defects and residual carrier doping ${ }^{46-48}$. In this regard, the low mobility of the surface states is a serious problem. However, recent work on TIs has shown a way to overcome this problem by studying transport in ultra thin samples, where a 12 times higher surface mobility as compared to the bulk can be achieved $^{49}$. It has been demonstrated that the large surface to volume ratio of $\mathrm{Bi}_{2} \mathrm{Se}_{3}$ thin films effectively suppresses bulk effects and enables probing of the electronic transport of the surface states ${ }^{50}$. There has been a rapid improvement in the sample quality coming along with a reduction of film thicknesses ${ }^{51}$. Relevant to the present study, the effect of the Zeeman exchange field in TIs is significant ${ }^{41,51}$ with a magnitude around $50 \mathrm{meV}$ due to the large effective g-factor. Also, recent experiments have found a hybridization gap in the ultrathin regime (less than $6 \mathrm{~nm})^{22,26}$ as well as quantum oscillations ${ }^{43}$. Therefore, it can be expected that the effects discussed before can be realized in quantum capacitance experiments in ultrathin films.

In conclusion, we have investigated the effects of Zeeman interaction and hybridization on the magnetocapacitance of a TI thin film in a perpendicular magnetic field. To this aim, we have obtained an analytic expression for the DOS incorporating both the Zeeman and hybridization contributions. It turns out that a combination of Zeeman interaction and hybridization breaks the electron-hole symmetry, resulting in significant effects on the magnetocapacitance at $\varepsilon_{F}$
$=0$. In addition, we have shown that in the presence of both Zeeman interaction and hybridization, the magnetocapacitance exhibits beating at low and level splitting at high perpendicular magnetic field. This behaviour is explained by interference of the $\mathrm{SdH}$ oscillations at the two frequencies induced by the splitting of the LLs. Let us comment on the relevance of our findings for experiments. The cyclotron energy for $B=1 \mathrm{~T}$ is $\hbar \omega=36 \mathrm{meV}$. Observation of the splitting of the LLs and the discussed consequences requires that the temperature is low and the system clean enough that disorder effects do not wash out the splitting. Temperature and disorder broadening thus must not reach the Zeeman or hybridization energies. For a tilted magnetic field with $\theta \rightarrow 90^{\circ}$ we find that the $\mathrm{SdH}$ oscillations are washed out.

1. Kane, C. L. \& Mele, E. J. Quantum Spin Hall Effect in Graphene. Phys. Rev. Lett. 95, 226801 (2005).

2. Bernevig, B. A. \& Zhang, S. C. Quantum Spin Hall Effect. Phys. Rev. Lett. 96, 106802 (2006).

3. Fu, L., Kane, C. L. \& Mele, E. J. Topological Insulators in Three Dimensions. Phys. Rev. Lett. 98, 106803 (2007).

4. Moore, J. E. \& Balents, L. Topological invariants of time-reversal-invariant band structures. Phys. Rev. B 75, 121306(R) (2007).

5. Fu, L. \& Kane, C. L. Topological insulators with inversion symmetry. Phys. Rev. B 76, 045302 (2007).

6. Qi, X. L., Hughes, T. L. \& Zhang, S. C. Topological field theory of time-reversal invariant insulators. Phys. Rev. B 78, 195424 (2008).

7. Roy, R. $Z_{2}$ classification of quantum spin Hall systems: An approach using timereversal invariance. Phys. Rev. B 79, 195321 (2009).

8. Qi, X. L. \& Zhang, S. C. Topological insulators and superconductors. Rev. Mod. Phys. 83, 1057-1110 (2011).

9. Hsieh, D. et al. A topological Dirac insulator in a quantum spin Hall phase. Nature 452, 970-974 (2008).

10. Chen, Y. L. et al. Experimental Realization of a Three-Dimensional Topological Insulator, $\mathrm{Bi}_{2} \mathrm{Te}_{3}$. Science 325, 178-181 (2009).

11. Hsieh, D. et al. A tunable topological insulator in the spin helical Dirac transport regime. Nature 460, 1101-1105 (2009).

12. Xia, Y. et al. Observation of a large-gap topological-insulator class with a single Dirac cone on the surface. Nat. Phys. 5, 398-402 (2009).

13. Burkov, A. A. \& Hawthorn, D. G. Spin and Charge Transport on the Surface of a Topological Insulator. Phys. Rev. Lett. 105, 066802 (2010).

14. Garate, I. \& Franz, M. Inverse Spin-Galvanic Effect in the Interface between a Topological Insulator and a Ferromagnet. Phys. Rev. Lett. 104, 146802 (2010).

15. Yokoyama, T., Zang, J. \& Nagaosa, N. Theoretical study of the dynamics of magnetization on the topological surface. Phys. Rev. B 81, 241410(R) (2010).

16. Hasan, M. Z. \& Kane, C. L. Colloquium: Topological insulators. Rev. Mod. Phys. 82, 3045-3067 (2010).

17. Alpichshev, Z. et al. STM Imaging of Electronic Waves on the Surface of $\mathrm{Bi}_{2} \mathrm{Te}_{3}$ : Topologically Protected Surface States and Hexagonal Warping Effects. Phys. Rev. Lett. 104, 016401 (2010).

18. Cheng, P. et al. Landau Quantization of Topological Surface States in $\mathrm{Bi}_{2} \mathrm{Se}_{3}$. Phys. Rev. Lett. 105, 076801 (2010).

19. Jiang, Y. et al. Landau Quantization and the Thickness Limit of Topological Insulator Thin Films of $\mathrm{Sb}_{2} \mathrm{Te}_{3}$. Phys. Rev. Lett. 108, 016401 (2012).

20. Liu, M. et al. Crossover between Weak Antilocalization and Weak Localization in a Magnetically Doped Topological Insulator. Phys. Rev. Lett. 108, 036805 (2012).

21. Song, C. L. et al. Gating the charge state of single Fe dopants in the topological insulator $\mathrm{Bi}_{2} \mathrm{Se}_{3}$ with a scanning tunneling microscope. Phys. Rev B 86, 045441 (2012).

22. Zhang, Y. et al. Crossover of the three-dimensional topological insulator $\mathrm{Bi}_{2} \mathrm{Se}_{3}$ to the two-dimensional limit. Nat. Phys. 6, 584-588 (2010).

23. Liu, C. X. et al. Oscillatory crossover from two-dimensional to three-dimensional topological insulators. Phys. Rev. B 81, 041307(R) (2010).

24. Yu, R., Zhang, W., Zhang, H. J., Zhang, S. C., Dai, X. \& Fang, Z. Quantized Anomalous Hall Effect in Magnetic Topological Insulators. Science 329, 61 (2010).

25. Linder, J., Yokoyama, T. \& Sudbø, A. Anomalous finite size effects on surface states in the topological insulator $\mathrm{Bi}_{2} \mathrm{Se}_{3}$. Phys. Rev. B 80, 205401 (2009).

26. Lu, H. Z., Shan, W. Y., Yao, W., Niu, Q. \& Shen, S. Q. Massive Dirac fermions and spin physics in an ultrathin film of topological insulator. Phys. Rev. B 81, 115407 (2010).

27. Zyuzin, A. A. \& Burkov, A. A. Thin topological insulator film in a perpendicular magnetic field. Phys. Rev. B 83, 195413 (2011).

28. Zyuzin, A. A., Hook, M. D. \& Burkov, A. A. Parallel magnetic field driven quantum phase transition in a thin topological insulator film. Phys. Rev. B 83, 245428 (2011).

29. Wang, Z., Fu, Z. G., Wang, S. X. \& Zhang, P. Magnetic quantum oscillations for the surface states of topological insulator $\mathrm{Bi}_{2} \mathrm{Se}_{3}$. Phys. Rev. B 82, 085429 (2010). 
30. Taskin, A. A. \& Ando, Y. Berry phase of nonideal Dirac fermions in topological insulators. Phys. Rev. B 84, 035301 (2011).

31. Mikitik, G. P. \& Sharlai, Y. V. Berry phase and the phase of the Shubnikov-de Haas oscillations in three-dimensional topological insulators. Phys. Rev. B 85, 033301 (2012).

32. Ilani, S., Donev, L. A. K., Kindermann, M. \& McEuen, P. L. Measurement of the quantum capacitance of interacting electrons in carbon nanotubes. Nat. Phys. 2, 687-691 (2006).

33. Guo, J., Yoon, Y. \& Ouyang, Y. Gate Electrostatics and Quantum Capacitance of Graphene Nanoribbons. Nano Lett. 7, 1935-1940 (2007).

34. Xia, J., Chen, F., Li, J. \& Tao, N. Measurement of the quantum capacitance of graphene. Nat. Nanotechnology 4, 505-509 (2009).

35. Giannazzo, F., Sonde, S., Raineri, V. \& Rimini, E. Screening Length and Quantum Capacitance in Graphene by Scanning Probe Microscopy. Nano Lett. 9, 23-29 (2009).

36. Ponomarenko, L. A. et al. Density of States and Zero Landau Level Probed through Capacitance of Graphene. Phys. Rev. Lett. 105, 136801 (2010).

37. Xu, H., Zhang, Z. \& Peng, L. M. Measurements and microscopic model of quantum capacitance in graphene. Appl. Phys. Lett. 98, 133122 (2011).

38. Fang, T., Konar, A., Xing, H. \& Jena, D. Carrier statistics and quantum capacitance of graphene sheets and ribbons. Appl. Phys. Lett. 91, 092109 (2007).

39. Fu, L. Hexagonal Warping Effects in the Surface States of the Topological Insulator $\mathrm{Bi}_{2} \mathrm{Te}_{3}$. Phys. Rev. Lett. 103, 266801 (2009).

40. Liu, C. X., Qi, X. L., Zhang, H. J., Dai, X., Fang, Z. \& Zhang, S. C. Model Hamiltonian for topological insulators. Phys. Rev. B 82, 045122 (2010).

41. Chen, Y. L. et al. Massive Dirac Fermion on the Surface of a Magnetically Doped Topological Insulator. Science 329, 659-662 (2010).

42. Cho, S., Butch, N. P., Paglione, J. \& Fuhrer, M. S. Insulating Behavior in Ultrathin Bismuth Selenide Field Effect Transistors. Nano Lett. 11, 1925-1927 (2011).

43. Taskin, A. A., Sasaki, S., Segawa, K. \& Ando, Y. Manifestation of Topological Protection in Transport Properties of Epitaxial $\mathrm{Bi}_{2} \mathrm{Se}_{3}$ Thin Films. Phys. Rev. Lett. 109, 066803 (2012).

44. Brüne, C. et al. Quantum Hall Effect from the Topological Surface States of Strained Bulk HgTe. Phys. Rev. Lett. 106, 126803 (2011).

45. Xiu, F. et al. Quantum Capacitance in Topological Insulators. Scientific Reports 2, $669(2012)$
46. Arakane, T. et al. Tunable Dirac cone in the topological insulator $\mathrm{Bi}_{2-x} \mathrm{Sb}_{x} \mathrm{Te}_{3-\gamma} \mathrm{Se}_{y}$. Nat. Commun. 3, 636 (2012).

47. Zhang, J. et al. Band structure engineering in $\left(\mathrm{Bi}_{1-x} \mathrm{Sb}_{x}\right)_{2} \mathrm{Te}_{3}$ ternary topological insulators. Nat. Commun. 2, 574 (2011).

48. Bianchi, M. et al. Coexistence of the topological state and a two-dimensional electron gas on the surface of $\mathrm{Bi}_{2} \mathrm{Se}_{3}$. Nat. Commun. 1, 128 (2010).

49. Qu, D. X., Hor, Y. S., Xiong, J., Cava, R. J. \& Ong, N. P. Quantum Oscillations and Hall Anomaly of Surface States in the Topological Insulator $\mathrm{Bi}_{2} \mathrm{Te}_{3}$. Science 329, 821-824 (2010).

50. Min, Y. et al. Quick, Controlled Synthesis of Ultrathin $\mathrm{Bi}_{2} \mathrm{Se}_{3}$ Nanodiscs and Nanosheets. J. Am. Chem. Soc. 134, 2872-2875 (2012)

51. Xu, S. Y. et al. arXiv:1206.2090.

\section{Acknowledgments}

K. Sabeeh would like to acknowledge the support of the Abdus Salam International Center for Theoretical Physics (ICTP) in Trieste, Italy through the Associate scheme where a part of this work was completed and the Higher Education Commission (HEC) of Pakistan for support through project No. 20-1484/R\&D/09.

\section{Author contributions}

M.T. performed the calculations. K.S. contributed to the calculations. M.T. and U.S. wrote the manuscript.

\section{Additional information}

Competing financial interests: The authors declare no competing financial interests.

License: This work is licensed under a Creative Commons

Attribution-NonCommercial-NoDerivs 3.0 Unported License. To view a copy of this license, visit http://creativecommons.org/licenses/by-nc-nd/3.0/

How to cite this article: Tahir, M., Sabeeh, K. \& Schwingenschlögl, U. Quantum capacitance of an ultrathin topological insulator film in a magnetic field. Sci. Rep. 3, 1261; DOI:10.1038/srep01261 (2013). 